\title{
Necroptosis in microglia contributes to neuroinflammation and retinal degeneration through TLR4 activation
}

\author{
Zijing Huang ${ }^{1,2}$, Tian Zhou ${ }^{1,2}$, Xiaowei Sun ${ }^{1}$, Yingfeng Zheng ${ }^{1}$, Bing Cheng ${ }^{1}$, Mei Li ${ }^{1}$, Xialin Liü ${ }^{\star, 1}$ and Chang He ${ }^{\star, 1}$
}

Inflammation has emerged to be a critical mechanism responsible for neural damage and neurodegenerative diseases. Microglia, the resident innate immune cells in retina, are implicated as principal components of the immunological insult to retinal neural cells. The involvement of microglia in retinal inflammation is complex and here we propose for the first time that necroptosis in microglia triggers neuroinflammation and exacerbates retinal neural damage and degeneration. We found microglia experienced receptorinteracting protein kinase 1 (RIP1)- and RIP3-dependent necroptosis not only in the retinal degenerative rd1 mice, but also in the acute retinal neural injury mice. The necroptotic microglia released various pro-inflammatory cytokines and chemokines, such as tumor necrosis factor- $\alpha$ and chemokine (C-C motif) ligand 2, which orchestrated the retinal inflammation. Importantly, necroptosis blockade using necrostatin-1 could suppress microglia-mediated inflammation, rescue retinal degeneration or prevent neural injury in vivo. Meanwhile, cultured microglia underwent RIP1/3-mediated necroptosis and the necroptotic microglia produced large amounts of pro-inflammatory cytokines in response to lipopolysaccharide or oxidative stress in vitro. Mechanically, TLR4 deficiency ameliorated microglia necroptosis with decreased expression levels of machinery molecules RIP1 and RIP3, and suppressed retinal inflammation, suggesting that TLR4 signaling was required in microglia necroptosis-mediated inflammation. Thus, we proposed that microglia experienced necroptosis through TLR4 activation, promoting an inflammatory response that serves to exacerbate considerable neural damage and degeneration. Necroptosis blockade therefore emerged as a novel therapeutic strategy for tempering microglia-mediated neuroinflammation and ameliorating neural injury and neurodegenerative diseases.

Cell Death and Differentiation (2018) 25, 180-189; do:10.1038/cdd.2017.141; published online 8 September 2017

Neuroinflammation contributes to neural damage and death and drives the progression and deterioration of multiple neurodegenerative diseases, such as Alzheimer's and Parkinson's diseases, and retinal degeneration. ${ }^{1-3}$ Proinflammatory cytokines and chemokines could induce neuron apoptosis and death directly, or further exacerbate neural damage indirectly by activating and recruiting immune cells. ${ }^{4,5}$ Manipulating neuroinflammation emerges as a promising strategy for neurodegenerative diseases as it has the potential to stop the ongoing of neural damage.

Neuroinflammation in central nervous system (CNS) is characterized by the activation of resident immune cells, known as microglia, which constitute the first defensive line in healthy brain and retina.,7 Microglia stay physically in a characteristic resting statue with high motility to survey their microenvironment for maintaining CNS homeostasis. Injury triggers retinal microglia to transform into an activated state rapidly, switching their function from patrolling to shielding of the injured sites. Emerging evidence suggests a prominent role of pathologically activated microglia in the initiation or aggravation of neurodegenerative diseases., 8 The activated microglia constantly secrete inflammatory mediators that can act on other cells to induce and amplify the uncontrolled inflammatory responses. However, the underlying mechanism of microglia-mediated inflammation remains unclear.

A newly defined necroptosis, a form of well-regulated necrosis executed by receptor-interacting protein kinase 1 (RIP1) and/or RIP3, is associated with an inflammatory reaction. ${ }^{10,11}$ Cellular necroptosis mediated by RIP $1 / 3$ is believed to be a crucial factor in triggering local inflammation in various diseases, such as graft cells in kidney and heart transplantation, ${ }^{12,13}$ Paneth cells in intestinal bowel disease and keratinocytes in skin inflammation. ${ }^{14,15}$ Necroptosis is different from apoptosis, which could efficiently clear the cell corpse and limit triggering of the innate immune system. Meanwhile, necroptosis exhibits distinct process from the cell death caused by necrosis, which is a passive decay of damaged cells and highly inflammatory. ${ }^{10,16}$ Increasing evidence suggests the pivotal role of necroptosis in inflammation, however, whether and how RIP1/3-mediated necroptosis contribute to the neuroinflammatory in CNS, particularly in retina, are not clear yet. Exploring the mechanism of necroptosis and its contribution to the neuroinflammatory pathogenesis may expand a new scope for CNS degenerative diseases.

In this study, we proposed that microglia necroptosis orchestrated the inflammation in retina and contributed to neural damage and degenerative diseases. We utilized the

\footnotetext{
${ }^{1}$ State Key Laboratory of Ophthalmology, Zhongshan Ophthalmic Center, Sun Yat-Sen University, Guangzhou, China

*Corresponding author: X Liu or C He, State Key Laboratory of Ophthalmology, Zhongshan Ophthalmic Center, Sun Yat-Sen University, Guangzhou 510060, China. Tel: +86 20 87330294; Fax: +86 20 87333271; E-mail: liux|28@mail.sysu.edu.cn or hech33@mail.sysu.edu.cn

${ }^{2}$ These authors contributed equally to this work.

Received 27.3.17; revised 09.7.17; accepted 24.7.17; Edited by N Bazan; published online 08.9.17
} 
a

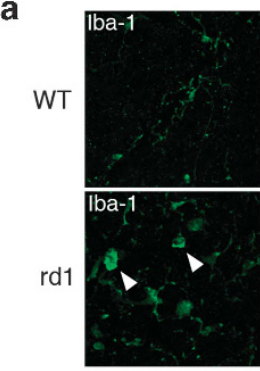

c

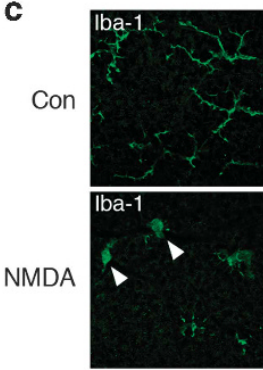

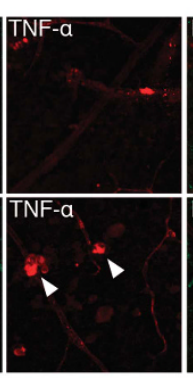
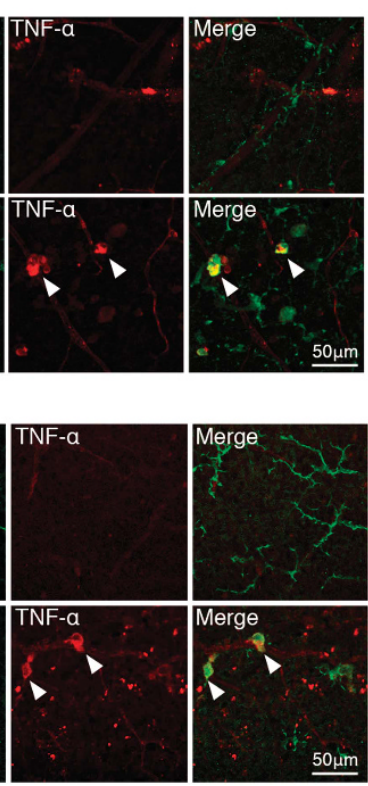

b

WT
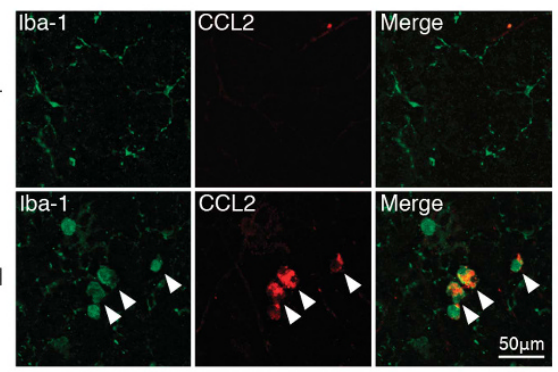

d
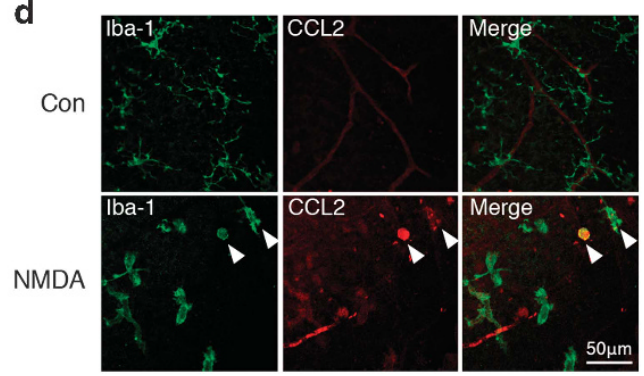

Figure 1 Increased TNF- $\alpha$ and CCL2 production from microglia in retinal degeneration and NMDA-induced injury mice. (a and $\mathbf{b}$ ) Retinae of rd1 and C57BL/6J control mice were used for flat-mount immunostaining at P14. Representative images showed that resting microglia in the control group featured with typical small cell bodies and long processes, and scarcely expressed TNF- $\alpha$ and CCL2. In contrast, the lba- $1^{+}$microglia in rd1 mice assumed activated phenotypes, presented with enlarged somas and short lamellipodia. In addition, a strong co-labeling of TNF- $\alpha$ or CCL2 with lba- $1^{+}$(white arrows) was observed in rd1 mice. (c and d) Adult C57BL/6J mice received a single intravitreal injection of NMDA to establish retinal neural injury, and vehicle (PBS) injection served as control. Retinae were harvested $24 \mathrm{~h}$ later for immunostaining. Similarly, lba- ${ }^{+}$microglia in control mice exhibited resting statue, whereas microglia in NMDA-insult mice displayed activated morphology and expressed abundant TNF- $\alpha$ and CCL2. All images were acquired by confocal microscopy (Carl Zeiss LSM710). At least six mice were used per group. The right retina from each mouse was cut into four pieces equally and two images were captured randomly in each quarter of the retina. Scale bar: $50 \mu \mathrm{m}$

retinal degeneration model of rd1 mice and established an acute retinal neural injury model by $N$-methyl-D-aspartate (NMDA) injection. Our results revealed that microglia underwent RIP1- and RIP3-mediated necroptosis and released cytokines and chemokines, such as tumor necrosis factor- $a$ (TNF- $a$ ) and chemokine (C-C motif) ligand 2 (CCL2), to initiate and/or sustain inflammatory responses, driving progression of neurodegenerative diseases.

\section{Results}

Pro-inflammatory cytokines and chemokines are produced by microglia during retinal injury and degeneration. Neuroinflammation has a vital role in neurodegeneration. TNF- $a$ and CCL2 are recognized to facilitate neuroinflammation and exacerbate neural injury in $\mathrm{CNS} .^{5,17}$ To evaluate the cellular source of these pro-inflammatory cytokines in the injured retinae, we utilized the retinal degeneration model of rd1 mice and established an acute retinal neural injury model by NMDA insults. The expression of TNF- $a$ and CCL2 were indeed increased significantly in the whole retina from either rd1 mice at P14 or acute neural injury mice $24 \mathrm{~h}$ after NMDA injection (Supplementary Figures S1a-c, and e), indicating the involvement of pro-inflammatory TNF- $a$ and CCL2 in the diseased retina. Other pro-inflammatory cytokines including IL-17, IL-1 $\beta$, IL-23 and IFN- $\gamma$ were also elevated in the retinae from rd1 or NMDA-damaged mice, further suggesting the presence of neuroinflammation associated with retinal neural injury (Supplementary Figures S1d and f).
As retinal microglia served as resident immune cells and a potent inducer of inflammatory responses in retina, we detected the expression of TNF- $a$ and CCL2 on $\mathrm{Iba}_{-1}{ }^{+}$ microglia. It was noted that TNF- $a$ and CCL2 were mainly located in activated microglia under pathological conditions, as characterized by large cell bodies with short and thick processes. In contrast, resting microglia with ramified processes in the normal retina showed low expression of these cytokines (Figures 1a-d). Together, these data demonstrated that microglia were activated and adopted pro-inflammatory phenotype with higher expression levels of TNF- $a$ and CCL2 in response to retinal degeneration and injury.

Microglia exhibit inflammatory phenotype under LPS stimulation or oxidative stress insults in vitro. We next investigated whether microglia displayed inflammatory phenotype in response to pathological stimuli in vitro. Murine BV2 microglial cells were cultured and identified using markers of Iba-1 and CD11b (Supplementary Figure S2). ${ }^{18,19}$ The classic inflammatory inducer of lipopolysaccharide (LPS) and hydrogen peroxide $\left(\mathrm{H}_{2} \mathrm{O}_{2}\right)$, which mimicked the oxidative stress insult during retinal degeneration, were applied, respectively. As expected, LPS administration increased the protein expression levels of TNF-a, CCL2 and IL-17 (Figure 2a), indicating the presence of a strong inflammation response. Similarly, their elevated expression levels were observed in BV2 cells exposed to $\mathrm{H}_{2} \mathrm{O}_{2}$ (Figure 2b). Inflammatory feature of $\mathrm{H}_{2} \mathrm{O}_{2}$-treated $\mathrm{BV} 2$ cells was further confirmed by immunofluorescence assays, which showed high levels of TNF- $a$ and IL-17 in BV2 cells under oxidative 
a
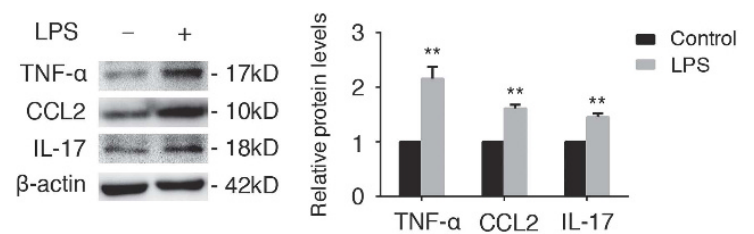

b

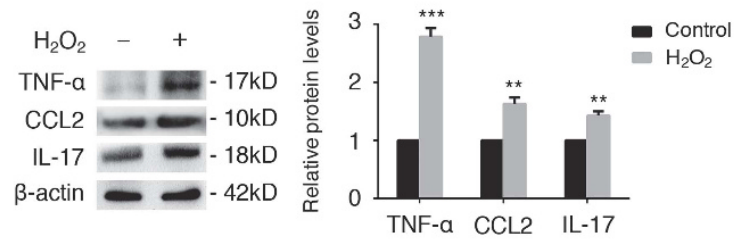

c

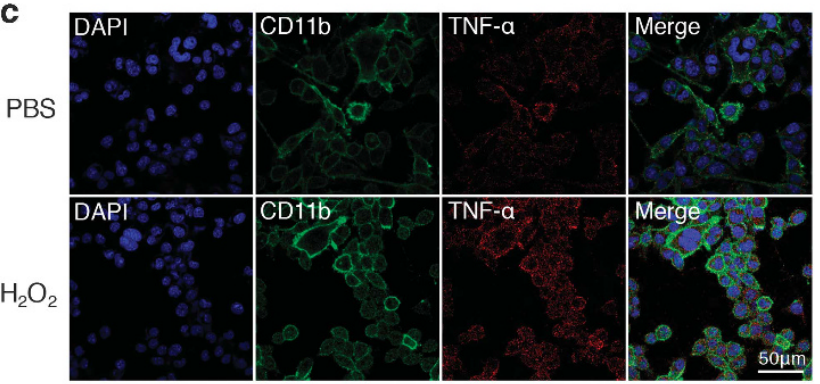

d

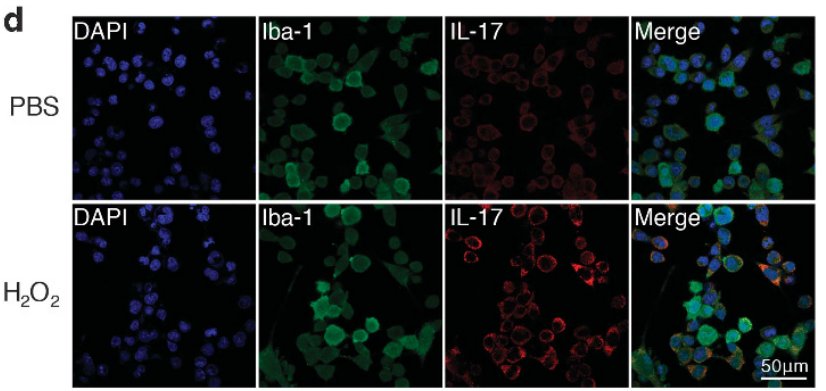

Figure 2 Inflammatory phenotype of microglia induced by LPS or oxidative stress in vitro. Cultured BV2 cells were stimulated with either LPS $(100 \mathrm{ng} / \mathrm{ml}), \mathrm{H}_{2} \mathrm{O}_{2}$ $(300 \mu \mathrm{M})$ or vehicle (PBS) for $24 \mathrm{~h}$, respectively. (a and $\mathbf{b})$ Cell lysates were prepared for western blotting. Increased expression levels of TNF- $\alpha$, CCL2 and IL-17 were observed in LPS- and $\mathrm{H}_{2} \mathrm{O}_{2}$-stimulated cells. $n=3$ independent cultures. (c and d) Immunostaining results showed increased expression of pro-inflammatory cytokines (TNF- $\alpha$ and IL-17) in $\mathrm{H}_{2} \mathrm{O}_{2}$-treated cells than PBS-treated ones. CD11b and Iba-1 were used as microglia markers. Eight images were collected randomly in each culture and the experiments were repeated at least three times. Scale bar: $50 \mu \mathrm{m}$. ${ }^{* *} P<0.01 ;{ }^{* \star \star} P<0.001$

stress than control (Figures $2 \mathrm{c}$ and d,Supplementary Figures $\mathrm{S} 1 \mathrm{~g}$ and $\mathrm{h}$ ). Taken together, these data showed that microglia contributed to the inflammation by releasing of proinflammatory cytokines under LPS stimulation or oxidative stress insults in vitro.

RIP1/3-dependent necroptosis in microglia contributes to retinal inflammation during retinal degeneration. As activated microglia were the main source of proinflammatory cytokines and the key mediator of inflammation response during retinal degeneration, we investigated the necroptosis profile of microglia in rd1 mice based on the novel idea of necroptosis-mediated inflammation. First, we detected the protein levels of the main mediators of necroptosis including RIP1 and RIP3, two key initiators of necroptosis, and their downstream protein MLKL. The WB results revealed that all these machineries of necroptosis were upregulated in the rd1 retina (Figure $3 a$ ). In addition, as RIP1 and RIP3 mediate cell death in a kinase activitydependent manner, we detected the kinase activation of RIP1 using IP assay and found that RIP1 kinase was significantly phosphorylated in rd1 retina (Figure 3b). These data implicate the involvement of RIP1/RIP3/MLKL-mediated necroptosis during retinal degeneration.

We next investigated the necroptosis feature of microglia by immunofluorescence. The results of rd1 retina exhibited the presence of TUNEL ${ }^{+}$cells, which were co-stained with lba-1, indicating that microglia experienced cell death during retinal degeneration (Figure 3c). Particularly, most of these cells also expressed high level of RIP3, a critical regulator of necroptosis (Figure 3c), indicating the microglia underwent cell death with necroptosis form. Of note, the $\mathrm{Iba}-1^{+} / \mathrm{RIP}^{+}$necroptotic microglia expressed TNF- $a$ and CCL2 simultaneously (Figures $3 d$ and $e$ ), suggesting that retinal microglia underwent necroptosis and released the pro-inflammatory cytokines.

Necrostatin-1 (Nec-1), a specific inhibitor of RIP1 kinase activity, was utilized to further verify the involvement of microglia necroptosis in neuroinflammation. Intravitreal injection of Nec-1 was performed at P9 in rd1 mice. At P14, we found decreased $\mathrm{TUNEL}^{+} / \mathrm{RIP}^{+}$necroptotic microglia (Figure 3c), further implicating that the RIP1 kinase-mediated necroptosis indeed occurred in microglia. In addition, as shown in the Figures $3 d$ and e, Nec-1 also reduced the expression of inflammatory cytokines of TNF- $a$ and CCL2 from RIP3+ microglia in the rd1 retina, indicating a prominent role of microglia necroptosis in the process of inflammation. In vitro, we found the expression levels of RIP1 and RIP3 increased significantly in response to $\mathrm{H}_{2} \mathrm{O}_{2}$, and blockade of necroptosis by Nec-1 impaired these two necroptotic machinery molecules (Supplementary Figures S3a and b), indicating that microglia would experience necroptosis under degenerative injury. Thus, our data may uncover a novel mechanism of microgliamediated inflammation that microglia experienced necroptosis and triggered neuroinflammation during retinal degeneration.

Necroptosis blockade using Nec-1 inhibits the retinal inflammation and rescues retinal degeneration. Blockade of necroptosis has shown protective effect in some inflammatory disease models, such as inflammatory bowel disease, dermatitis, pancreatitis, and virus- and alcohol-induced hepatitis. ${ }^{16}$ We next investigated the effect of Nec-1 on neuroinflammation in the degenerative retina. The western blot results confirmed the inhibition of RIP1 and RIP3 expression after $\mathrm{Nec}-1$ intravitreal injection in rd1 mice (Figure 4a). Concomitantly, the mRNA and protein expression of TNF- $a$ and CCL2 reduced after Nec-1 treatment (Figures 4b-d). In vitro data also revealed that not only RIP1 and RIP3, but also the pro-inflammatory cytokines decreased after Nec-1 administration (Supplementary Figures S3c and d).

To evaluate the effect of Nec-1 on retinal neural function, we performed the electroretinography (ERG) to measure the electrical potential change of the retina in response to light stimulation. The results revealed significant improvements of both a-wave and b-wave amplitudes, which represented the 


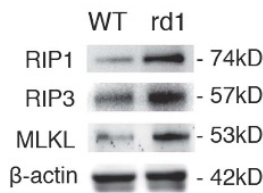

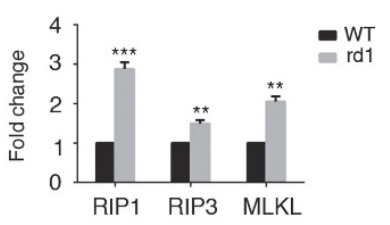

b
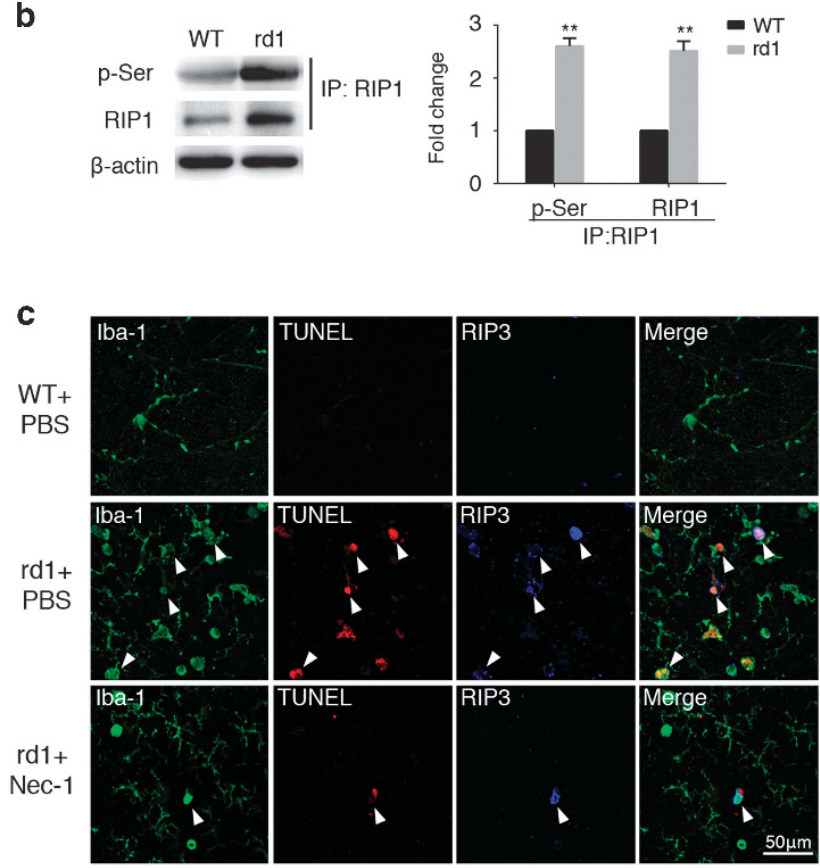

d
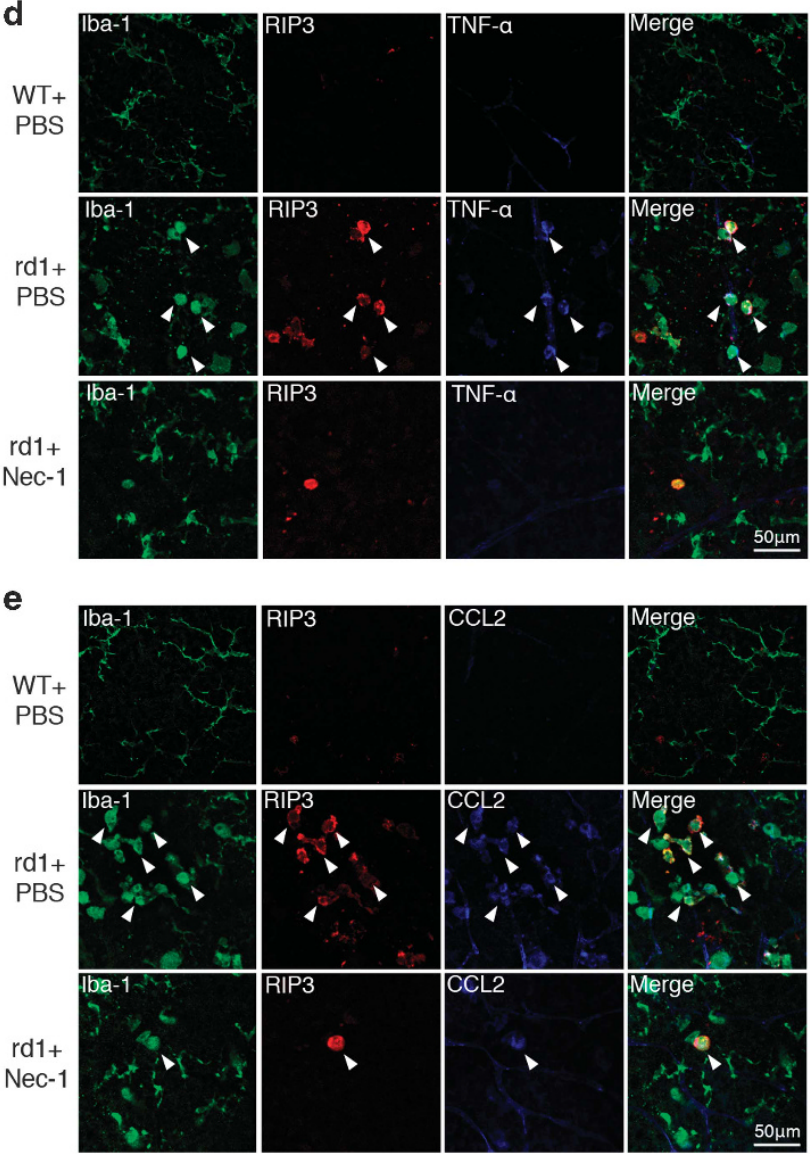

Figure 3 Microglia necroptosis triggered the inflammatory process in rd1 mice. Retinae of rd1 mice at P14 were extracted and analyzed in a-e. (a) Western blotting showed increased expression levels of RIP1, RIP3 and MLKL in retinae of rd1 mice. The RIP1 in rd1 was found to be up almost threefold more than that of same age C57 mice. (b) Immunoprecipitation (IP) followed by immunoblotting (IB) showed RIP1 activation in rd1 retinae. (c-e) Nec-1 was administrated in rd1 mice by intravitreal injection at P9 and the retinae at P14 were extracted for further analysis. Same age of PBS-treated C57 mice and PBS-treated rd1 mice were used as controls. (c) Compared with PBS-treated WT retina, PBS-treated rd1 retina exhibited more TUNEL-positive cells co-labeled with Iba-1 and elevated level of specific necroptosis marker RIP3, indicating the necroptosis of microglia in rd1. Nec-1 treatment markedly reduced TUNEL cells and RIP3 expression on microglia. ( $d$ and e) The lba- $1^{+}$microglia in PBS-treated rd1 mice expressed increased levels of RIP3 as well as inflammatory factors (TNF- $\alpha$ or CCL2) in comparison with PBS-treated C57 mice. Of note, lower expression of RIP3 and TNF- $\alpha$ or CCL2 was observed in the Nec-1-treated retina compared with PBS-treated rd1 mice. (a and $\mathbf{b}) n=4$ retinae per group. ${ }^{* *} P<0.01 ;{ }^{* *} P<0.001$; ns: no significance. (c-e) $n=6$ retinae per group and eight images per retina. Scale bar: $50 \mu \mathrm{m}$

neural signals derived from photoreceptors and second-order neuron in the retina, in Nec-1-treated mice at different light intensities (3.0 and $10.0 \mathrm{~cd} \mathrm{~s} / \mathrm{m}^{2}$ ) after dark-adapted condition (Figure 4f). Besides, increased thicknesses of the inner and outer nuclear layers were observed in the Nec-1-treated group (Figure $4 \mathrm{e}$ ), indicating the amelioration of retinal degeneration. In addition, the amount of apoptotic photoreceptors labeled with TUNEL was markedly reduced in Nec-1 group than the PBS controls (Figure 4g). These results showed that Nec-1 could inhibit necroptosis-associated inflammation and rescue the degenerative retina in rd1 mice.

Microglia undergo necroptosis and induce proinflammatory cytokines releasing in NMDA-induced retinal neural injury. Not only retinal degeneration but also acute neural injury after NMDA insults are often associated with neuroinflammation. ${ }^{20}$ We next evaluated whether necroptosis was required in microglia-mediated inflammation in the NMDA-induced retinal injury model. The immunostaining results showed that NMDA administration increased the expression of RIP3 and TNF- $a$ on the $\mathrm{Iba}-1^{+}$microglia, indicating that the activated microglia underwent necroptosis and expressed TNF- $a$ in response to NMDA injury. The inhibition of necroptosis using Nec-1 not only reduced the RIP3 expression, but also TNF-a, further suggesting that the RIP3-mediated necroptosis contributed to TNF-a release from microglia (Figure 5a). Similarly, NMDA induced increased expression levels of RIP3 and CCL2, whereas Nec-1 prevented microglia from experiencing RIP3-mediated necroptosis and releasing CCL2 (Figure 5b). The western blot results also revealed that NMDA insults triggered higher expression of RIP1, RIP3 and MLKL in the retina compared with PBS control group (Figure $5 \mathrm{c}$ ). Taken together, these results suggested that microglia experienced necroptosis in response to acute neural insults, accompanied by the release of pro-inflammatory cytokines.

We further detected the effect of Nec-1 on retinal inflammation and acute injury. Nec-1 was injected intravitreally with NMDA simultaneously, $24 \mathrm{~h}$ later the retinae were collected for further analysis. The western blot results revealed that $\mathrm{Nec}-1$ 
a

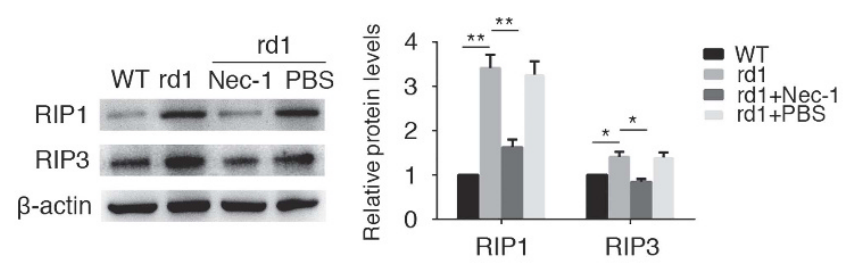

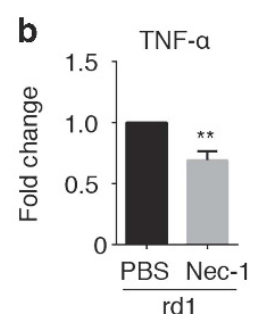

e

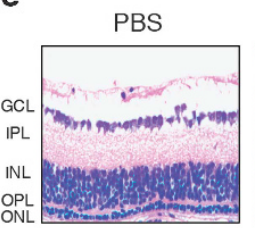

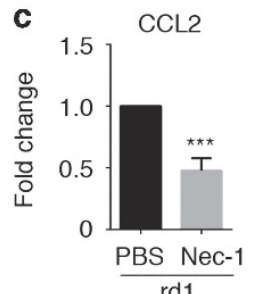

d

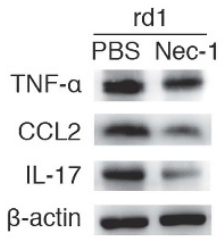

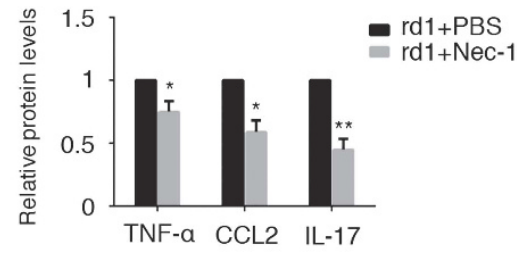

$\mathbf{f}$
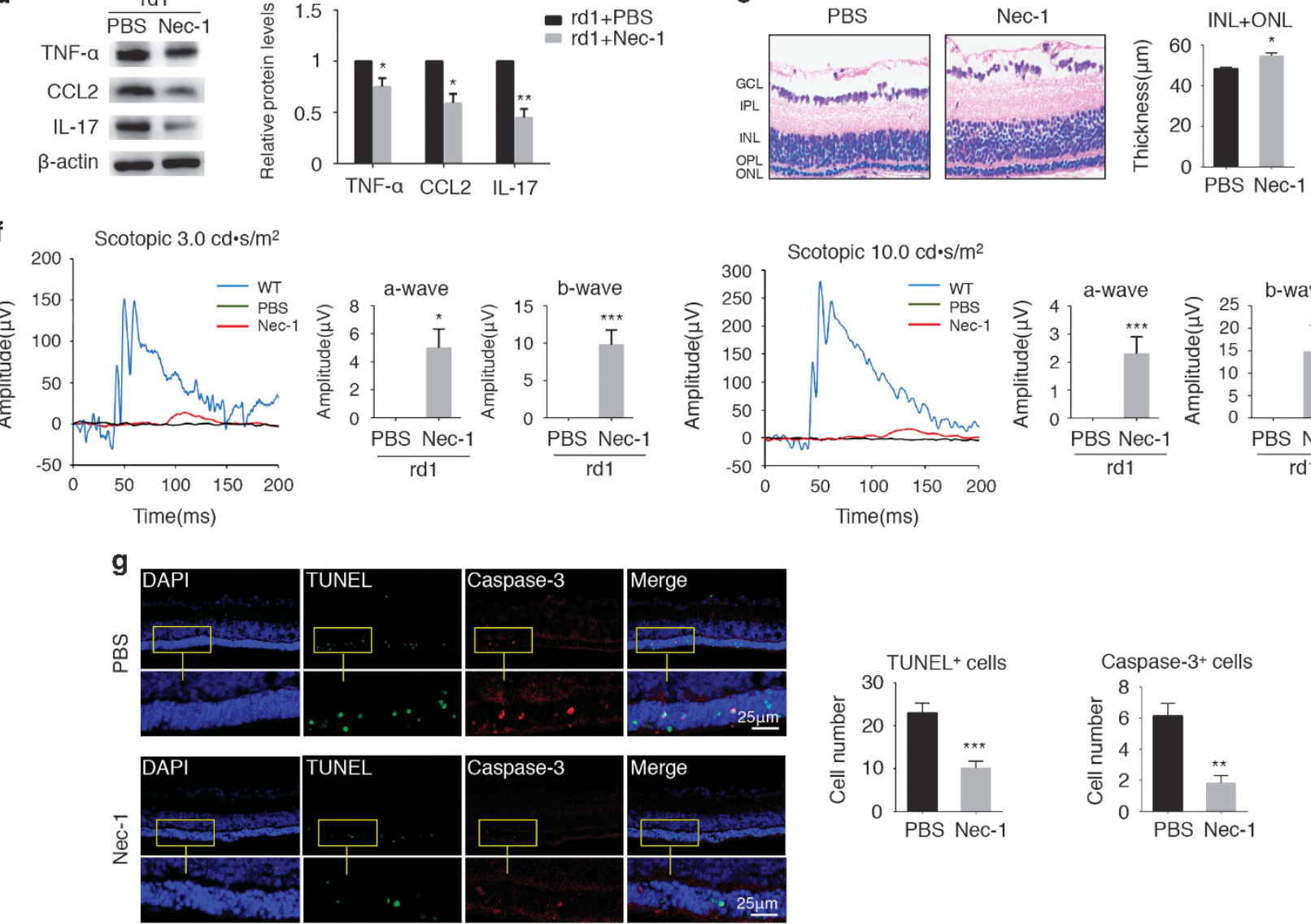

Figure 4 Necroptosis blockade attenuated retinal inflammation and rescued degeneration in rd1 mice. Nec-1 was injected intravitreally at P9 in rd1 mice and retina were collected and analyzed at P14. (a) Western blotting revealed elevated levels of necroptotic mediators (RIP1 and RIP3) in the rd1 retinae comparing with C57 ones. Reduced expression of RIP1 and RIP3 was observed in the rd1 after Nec-1 treatment. (b-d) Nec-1 significantly downregulated expression of TNF- $\alpha$ and CCL2 at both RNA and protein levels. (e) Retinal neural function was measured by ERG at P14. ERG was performed using single-flash recordings at two light intensities of 3.0 and $10.0 \mathrm{scotopic} c d \mathrm{~s} / \mathrm{m}^{2}$, respectively. C57 mice were used as normal control. No response was detected in PBS-treated rd1 mice at P14. After Nec-1 injection, significant improvements of both a-wave and b-wave amplitudes were observed. $n=8$ eyes per group. (f) Histologic alterations of the retinae were evaluated by H\&E staining on retinal paraffin sections. Nec-1-treated rd 1 mice exhibited an increase in the thickness of the inner and outer nuclear layers in comparison with the PBS-treated ones. IPL, inner plexiform layer; OPL, outer plexiform layer; ONL:, outer nuclear layer. $n=6$ eyes and six microscope fields per eye (g) Confocal microscopy showed that the number of TUNEL ${ }^{+}$and cleaved-caspase- $3^{+}$cells in the outer nuclear layer was decreased after Nec-1 administration compared with PBS treatment, indicating Nec-1 prevented the photoreceptors from apoptosis. For each panel, images below showed higher magnification of the selected areas of the above images respectively. $n=6$ eyes and six microscope fields per eye for statistical analysis. Scale bar: $25 \mu \mathrm{m}$. ${ }^{*} P<0.05 ;{ }^{* *} P<0.01 ;{ }^{* *} P<0.001$

could suppress both RIP1 and RIP3 expression, especially RIP3 in the retinae following acute NMDA insults (Figure 6a). Concomitantly, Nec-1 decreased the expression level of proinflammatory cytokines including TNF- $a$, CCL2 and IL-17, indicating that blockade of necroptosis could halt retinal inflammation (Figure 6b). As NMDA induced neurotoxicity in retinal neural cells, particularly the ganglion cells, we detected the rescue effect of Nec-1 on retinal cell death using TUNEL assays. As expected, NMDA induced significant cell death in the ganglion cell layer (GCL) and inner nuclear layer (INL) in the retina, whereas Nec-1 application reduced the number of apoptotic neural cells in these layers (Figure 6c). Consistently, histopathological alteration measured by H\&E staining showed more cellular nucleus in the GCL layer in the Nec-1 treatment group compared with the PBS group (Figure 6d). These data thus demonstrated that $\mathrm{Nec}-1$ could suppress microglia necroptosis and inflammation, and attenuate acute retinal damage induced by NMDA. 
a

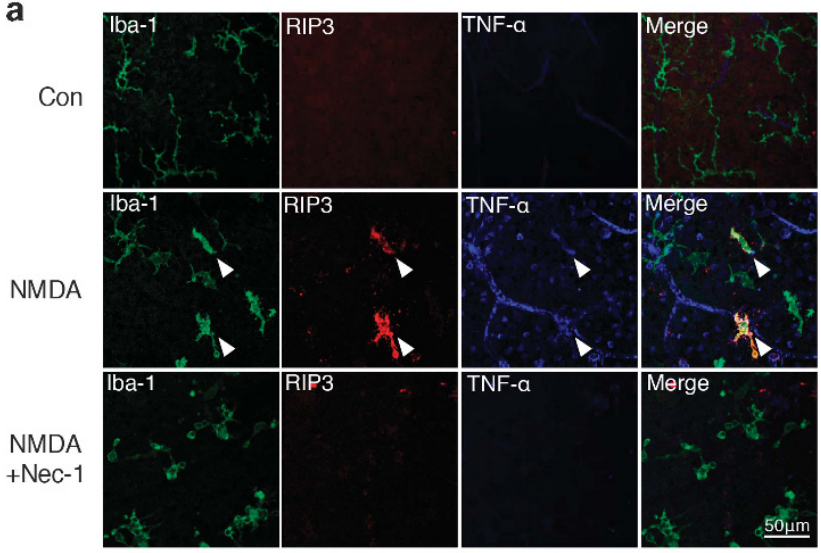

b
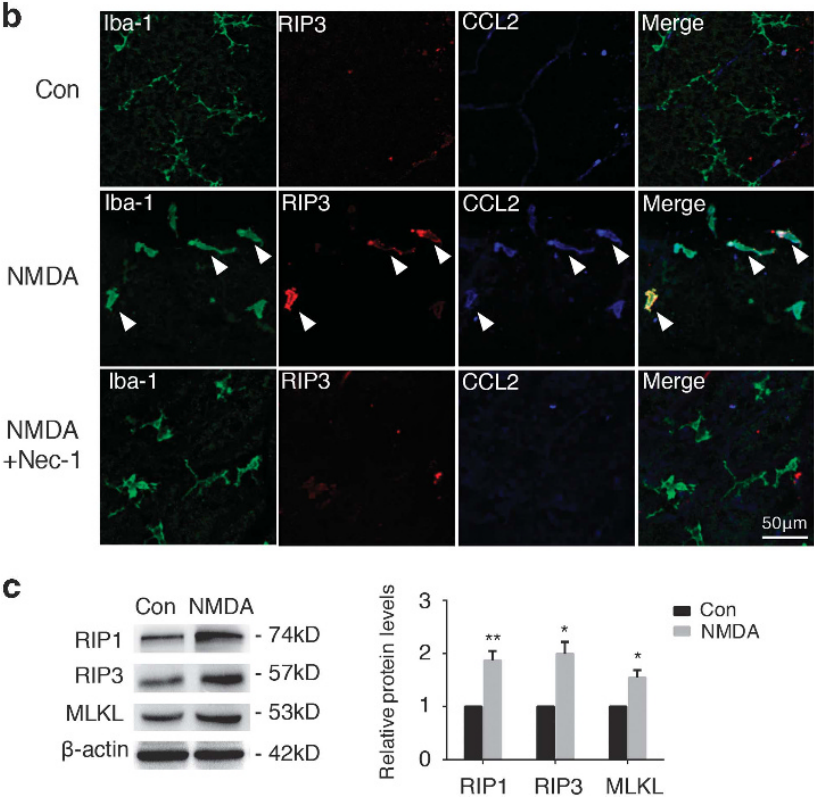

Figure 5 Involvement of microglia necroptosis in the inflammatory process after acute retinal injury. For Nec-1 treatment in the NMDA-induced retinal injury mice model, NMDA and Nec-1 resolved in $1 \mu \mathrm{l}$ PBS were injected into the vitreous of 8week-old mice. Retinae were extracted $24 \mathrm{~h}$ later. (a and $\mathbf{b}$ ) Immunostaining on retinal flat-mounts showed increased levels of RIP 3 , as well as inflammatory factors (TNF- $\alpha$, $\mathrm{CCL} 2)$ on $\mathrm{Iba}-1^{+}$microglia after NMDA administration, whereas Nec-1 could decrease their expression, indicating the role of microglia necroptosis in the inflammation response. $n=6$ retinae per group. Scale bar: $50 \mu \mathrm{m}$. (c) Elevated protein levels of RIP1, RIP3, and their downstream MLKL from western blotting results confirmed the presence of necroptosis after NMDA damage. $n=4$ retinae per group. ${ }^{*} P<0.05 ;{ }^{*} P<0.01$

TLR4 activation, rather than TLR2, contributes to microglia necroptosis-mediated inflammation in vivo and in vitro. The TLRs are considered to induce necrosome formation and initiate the necroptotic signaling. ${ }^{21,22}$ We next investigated the potential role of TLR2 and TLR4, the main surface receptors on microglia, in necroptosis and inflammation. Increased expression level of TLR2 and TLR4 on


suggesting the involvement of TLR2/4 activation during retinal neural injury (Figures $7 a$ and b). Furthermore, TLR2 and TLR4 knockout mice were used to establish the NMDAinduced retinal neural injury model. We found that in WT mice most Iba- $1^{+} \mathrm{RIP}^{+}$necroptotic microglia released TNF-a. However, TLR4 deficiency exhibited absence of RIP3 and TNF- $a$ in the microglia, whereas TLR2 deficiency just mildly reduced the RIP3 and TNF- $a$ expression on $\mathrm{Iba}-1^{+}$microglia (Figure 7c). Similarly, TLR4 deficiency displayed no staining of RIP3 and CCL2 in the microglia at all, whereas TLR2 deficiency exhibited almost equal level of RIP3 and CCL2 compared with the WT mice (Figure 7d), indicating that TLR4, rather than TLR2, is required in microglia necroptosis and inflammation in response to acute injury. The western blot results also revealed that TLR4 deficiency significantly reduced the expression levels of RIP1 and RIP3, whereas there were almost no changes of RIP1 and RIP3 levels in the $\mathrm{TLR}^{-1-}$ group compared with WT retinae (Figures 7e and g). Accordingly, TLR4 knockout mice produced less inflammatory mediators (TNF- $a$, CCL2 and IL-17) in NMDA model, as TLR2 deficiency had trivial impact on the expression levels of these cytokines (Figures $7 f$ and $g$ ). In vitro, TLR4 knockdown by siRNA in cultured BV2 cells suppressed the expression levels of RIP1 and RIP3. However, TLR2 knockdown failed to achieve the same effect (Figures $7 \mathrm{~h}$ and $\mathrm{j}$ ). Consistently, TLR4 knockdown led to much lower expression of inflammatory cytokines (TNF- $a$, CCL2 and IL-17), whereas TLR2 knockdown exhibited just a mild influence on them (Figures $7 \mathrm{i}$ and $\mathrm{j}$ ). Altogether, these data provided evidence for the requirement of TLR4 but not TLR2 as a key regulator in necroptosis signaling.

\section{Discussion}

Neuroinflammation is a prominent feature shared by various neural injuries and degenerative diseases. We first proposed the involvement of necroptosis-mediated inflammation in different retinal neural injury models. Retinal microglia, the essential resident immune cell in local inflammatory procession, experienced RIP1- and RIP3-dependent necroptosis and released pro-inflammatory cytokines and chemokine in vivo and in vitro. Necroptosis blockade using Nec-1 retarded the production of inflammatory mediators and prevented the retinal neurons from injury and degeneration, providing a novel target for suppressing inflammation and ameliorating neural injuries and degenerative diseases.

Traditionally, brain and retina were considered as immune privilege organs owing to the existence of blood-brain barrier and blood-retina barrier (BRB), which limited inflammatory invasion. However, emerging studies have now demonstrated that microglia served as major resident immune cells and key factors mediating the inflammatory process during retinal injuries. ${ }^{9,23,24}$ In this study, retinal microglia readily underwent necroptosis in response to impairments and increased the production of cytokines, such as TNF- $a$, IL-17, CCL2 and so on, initiating and promoting inflammatory responses. As previous studies reported, TNF- $a$ facilitated CD95L-induced neuron apoptosis and CCL2 could accelerate beta-amyloid pathology and induce deficits in memory function of $A D$ mouse. ${ }^{25,26}$ Necroptosis blockade could suppress the expression of these inflammatory mediators and remit neural apoptosis in this study, further corroborating the hypothesis of pro-inflammatory and detrimental roles of necroptotic microglia in different diseased models. Interestingly, we found 
a

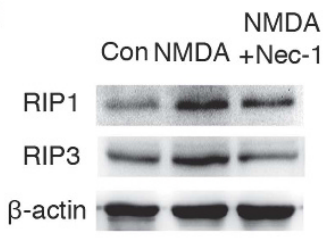

b

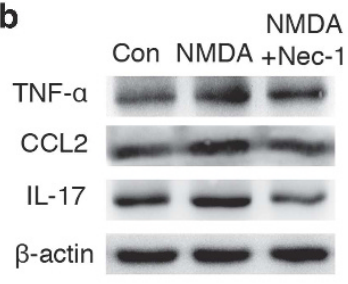

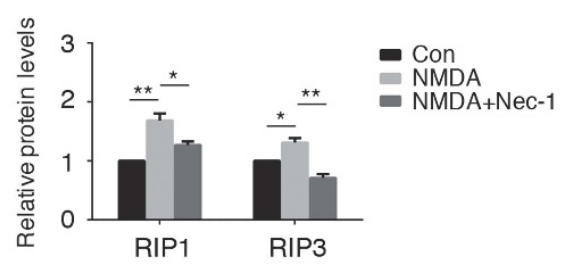

c
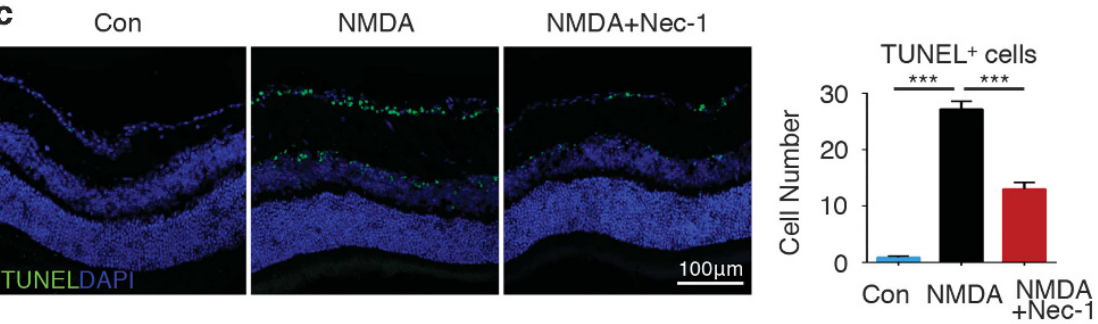

d Con

NMDA

NMDA+Nec-1
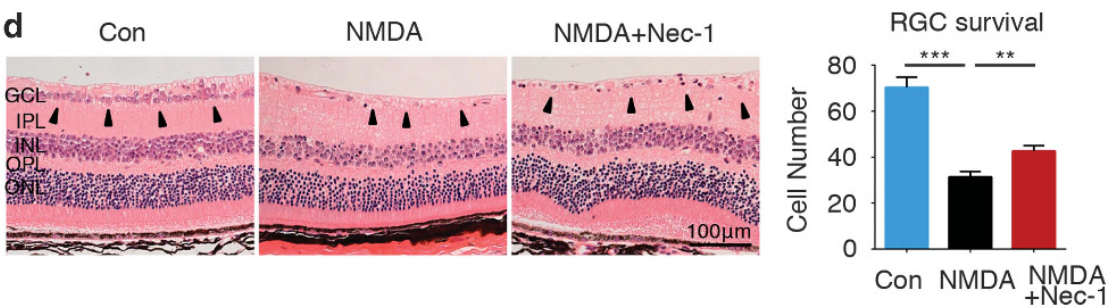

Figure 6 Anti-inflammatory and neuroprotective effect of Nec-1 in NMDA-induced neural injury. (a and b) Western blot showed that Nec-1 successfully downregulated necroptosis kinases (RIP1 and RIP3), as well as inflammatory cytokines (TNF- $\alpha$, CCL2 and IL-17). $n=4$ retinae per group. (c) Nec-1 treatment reduced the number of apoptotic neuronal cells (green) in the GCL and INL after NMDA injury measured by TUNEL assay. (d) H\&E staining showed that more retinal ganglion cells remained after Nec- 1 injection. $n=6$ eyes and six microscope fields per eye. Scale bar: $100 \mu \mathrm{m} .{ }^{\star} P<0.05 ;{ }^{\star \star} P<0.01 ;{ }^{\star \star *} P<0.001$

necroptotic microglia assumed an ameboid shape, which was the characteristic morphology of classical activated microglia, indicating that the activated microglia were partly fated to undergo necroptosis and induced the inflammation responses through necroptosis. Microglia necroptosis mostly occurred in the inflamed sites of the injured or degenerative retinae and supplemented another critical component of inflammation in the neural retinae besides activation, further suggesting that necroptosis orchestrated the neuroinflammation.

It is considered that the activation of microglia in response to neural insults induced BRB broken and circulating macrophages could migrate into the retina through damaged BRB during neuroinflammation. ${ }^{27}$ Many studies often used the term of microglia/macrophages to refer to these cells. Although a novel specific marker for microglia revealed in recent studies may help to confirm a prominent role of microglia in retinal degeneration, ${ }^{28}$ it would be interesting to determine whether macrophages also have a critical role despite its lower number compared with microglia. Furthermore, the similarity and difference between these microglia/macrophages, and their interchangeable functions remain to be further investigated.
Actually, the RIPs were reported to have many functions, including modulation of cell survival and death, the activation of cytokine production, etc. ${ }^{29}$ RIP1 and RIP3 induced cell death in a kinase activity-dependent manner, whereas their non-death functions are scaffold dependent and kinase activity disposable. ${ }^{29-31}$ In this study, we found RIP1 kinase was phosphorylated in rd1 mice, indicating RIP1 was inclined to induce cell death at the setting of neuroinflammatory and degenerative retinae. Nec-1 is a commercially available small molecule, which acts as an ATP competitive inhibitor that binds an inactive conformation of RIP1. It could block the interaction between RIP1 and RIP3 and inhibit the formation of necroptotic complex. ${ }^{32}$ In this study, we found Nec-1 treatment not only reduced the expression levels of RIP1, RIP3 and MLKL, but also significantly suppressed TUNEL staining on microglia, indicating the RIP1-RIP3-MLKL pathway was required in microglia necroptosis and could be blocked by Nec-1.

TLRs have been reported to display potential effects on stimulating RIP3-dependent necroptosis in the presence of a pan-caspase inhibitor. ${ }^{33}$ Among TLRs, TLR2 and TLR4 have been found to express abundantly on microglia during inflammation. ${ }^{34}$ In this study, we found TLR4-deficient mice 
a
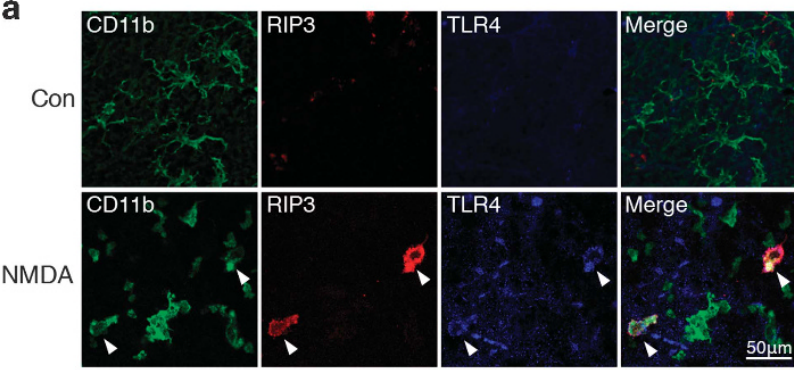

b

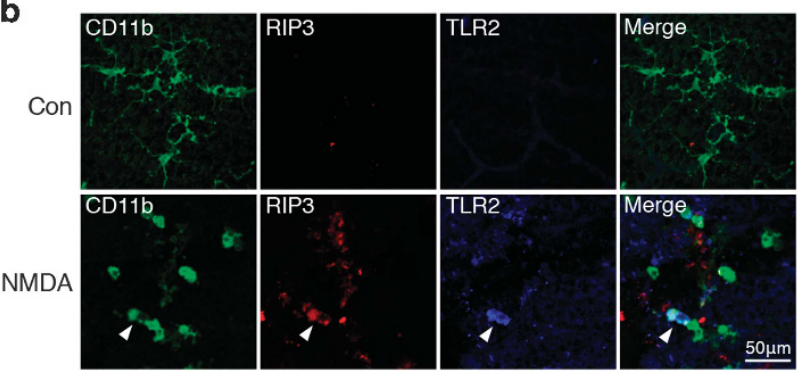

C

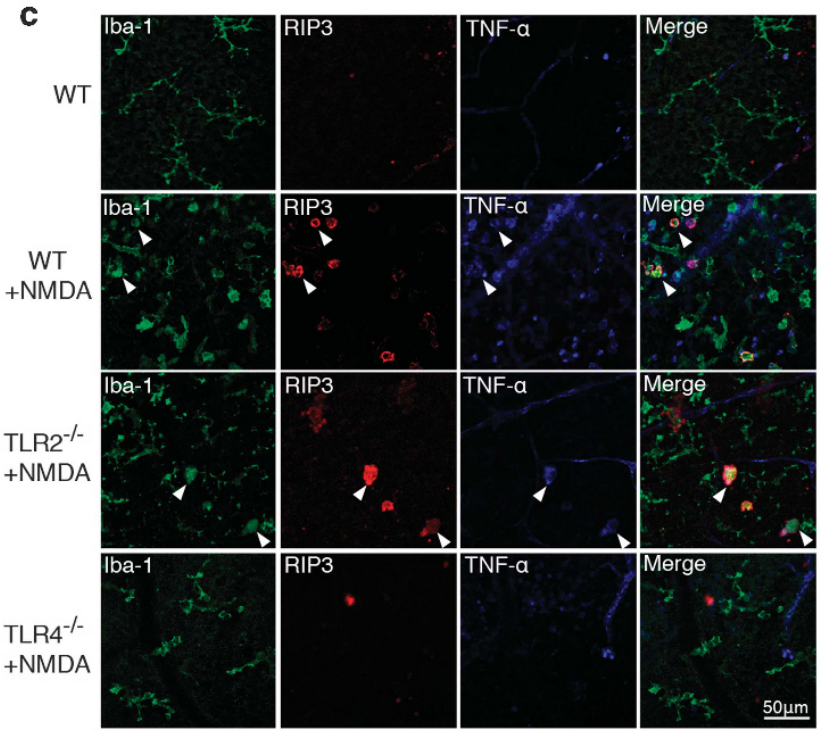

d

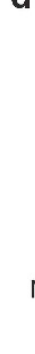

d

TLR2 $^{-/}$
+NMDA
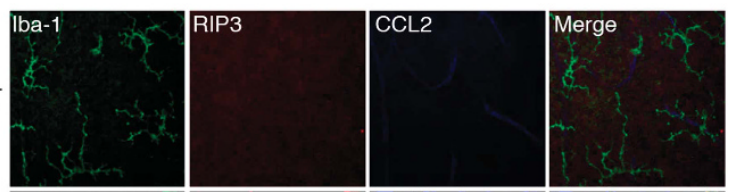

WT+
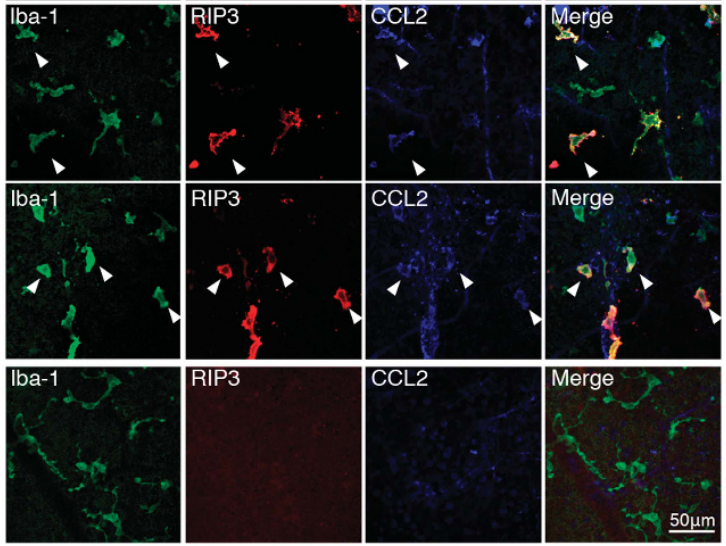

Merge
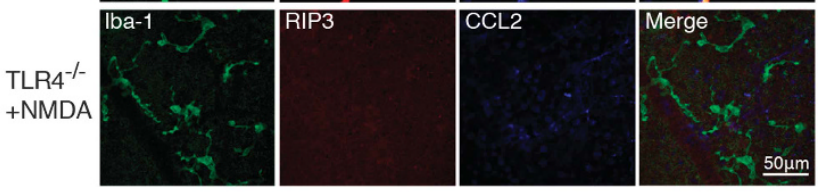

e
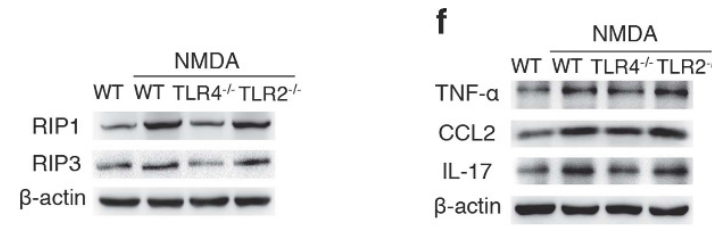

g

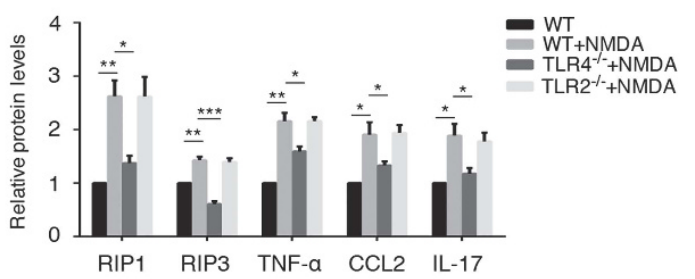

h
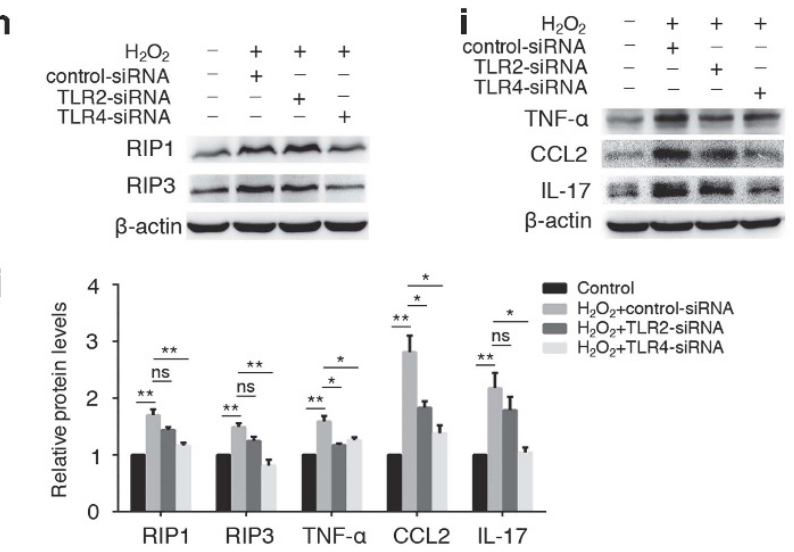

Figure 7 TLR4 activation rather than TLR2 was required in microglia necroptosis and inflammation. NMDA injury model was established on TLR2 ${ }^{-1-}$ and TLR4 ${ }^{-1-}$ mice. (a and b) Immunostaining on retinal flat-mounts showed distinct TLR2 and TLR4 activation in CD11b microglia from WT-NMDA mice (white arrows). (c and d) TLR4 knockout mice showed little RIP3 expression and Iba- $1^{+}$microglia co-labeled with either TNF- $\alpha$ or CCL2 was almost absent compared with WT-NMDA mice (white arrows), whereas in TLR2deficient mice, Iba- $1^{+}$microglia displayed almost similar expression levels of RIP3, TNF- $\alpha$ and CCL2. $n=6$ retinae and eight images per retina. (e) Western blot revealed that RIP1 and RIP3 expression levels were reduced in TLR4 ${ }^{-1-}$ but not TLR2 ${ }^{-1-}$ mice after NMDA damage. (f) TLR4 knockout mice exhibited significantly impaired production of inflammatory cytokines (TNF- $\alpha$, CCL2 and IL-17), whereas TLR2 deficiency showed nearly equal expression to WT mice. (g) Statistical analysis of western blotting are shown in e and f. $n=4$ retinae per group. (h) In vitro, BV2 cells transfected with either TLR2-siRNA, TLR4-siRNA, or control-siRNA were then stimulated with $\mathrm{H}_{2} \mathrm{O}_{2}$. Western blot showed decreased RIP1 and RIP3 expression in TLR4 rather than TLR2 knockdown cells. (i) Levels of inflammatory factors including IL-17, TNF- $\alpha$ and CCL2 were also significantly downregulated in TLR4-siRNA group compared with TLR2-siRNA and control-siRNA. (j) Statistical analysis of western blotting shown in $\mathbf{h}$ and $\mathbf{i}$. $n=4$ retinae per group. ${ }^{*} P<0.05 ;{ }^{* *} P<0.01 ;{ }^{* *} P<0.001 ;$ ns: no significance 
presented reduced expression of RIP1 and RIP3 following NMDA insults, and TLR4 knockdown in cultured microglia induced little RIP1/3 expression, indicating TLR4 activation was required in necroptotic signaling. Indeed, TLR4 could develop an endosome platform recruiting a cytosolic adaptor TRIF, which contains a RHIM domain for facilitating its interaction with RIP1 and RIP3. ${ }^{22}$ Although TLR2 has not been reported to induce necroptosis directly, TLR2 activation by Pam3CysK ligand formed an auto- or paracrine TNF production loop via a CD36-dependent mechanism, sensitizing cells to TNF-induced death by RIP1-dependent necroptosis. ${ }^{11}$ In this study, our findings confirmed the abundant expression of TLR2 on microglia during retinal inflammation. However, silence of TLR2 function failed to prevent microglia from necroptosis and was not sufficient to interdict the productions of TNF- $a$ and CCL2, indicating that TLR2 may not participate in microglia necroptosis. Thus, we characterized a potential mechanism that TLR4 activation contributed to necroptosis of microglia in response to neural injury.

Although the primary insult of many neurodegenerative diseases may not be inflammation, the immune cell-mediated inflammatory processes could urge the progression of retinal injuries and neurodegenerative diseases. Therapeutic approaches targeting immune cells to suppress inflammation have gained breakthroughs in recent advanced studies. Our results revealed that necroptosis in microglia induced and amplified inflammatory processes, accelerating neuronal damage in retina. Targeting this process may lead to a quantum leap in inflammation regulation, which is a prime therapeutic strategy for neural injuries and neurodegenerative diseases.

\begin{abstract}
Materials and Methods
Animals and disease models. Age-matched C57BL/6J mice (Guangzhou University of Chinese Medicine, Guangzhou, China), rd1 mice (pde6 $\beta^{\text {rd1 }}$ ) (Beijing Vital River Laboratory Animal Technology, Beijing, China), TLR2 ${ }^{-1-}$ and TLR4 ${ }^{-1-}$ mice (Model Animal Research Center of Nanjing University, Nanjing, China) were maintained in a specific pathogen-free facility in Animal Laboratories of Zhongshan Ophthalmic Center and were treated in accordance with the principles and procedures approved by the Institutional Animal Care and Use Committee of Zhongshan Ophthalmic Center, Sun Yat-Sen University, Guangzhou, China. NMDAinduced acute retina injury model was established according to previous studies. ${ }^{35}$ In brief, 8-week-old C57BL/6J mice or TLR2 ${ }^{-1-}$ and TLR4 ${ }^{-1-}$ mice were injected intravitreally with NMDA (40 nmol in $1 \mu \mathrm{l})$ after being anesthetized. Twenty-four hours after NMDA administration, the retinae were extracted for further analysis. For Nec-1 treatment, $1 \mu \mathrm{l} \mathrm{Nec-1} \mathrm{(Sigma} \mathrm{Chemical} \mathrm{Co.,} \mathrm{St.} \mathrm{Louis,} \mathrm{MO,} \mathrm{USA;} 1.0 \mathrm{mM}$, soluted in $30 \%$ [v/v] DMSO in PBS) or control vehicle (same resolvent) were injected into the vitreous using a 33-gauge needle in the right eye. The $\mathrm{rd} 1$ mice were treated with Nec-1 intravitreally at P9 and killed at P14. In the NMDA injury model, NMDA together with $\mathrm{Nec}-1$ ( $1 \mu \mathrm{l}$ in total) was injected intravitreally at the same time. Mice with visible surgical complication, such as retinal hemorrhage and retinal detachment, were excluded from the study.
\end{abstract}

Electroretinogram. Following 12-h dark adaptation, rd1 mice at P14 were anesthetized using $4.3 \%$ chloral hydrate $(10 \mathrm{ml} / \mathrm{kg}$ ) by intraperitoneal injection. Then, the pupil was dilated with $0.5 \%$ tropicamide eye drops and body temperature was maintained at $37^{\circ} \mathrm{C}$ through a heating pad. Two gold plated loop electrodes contacting the corneal surface were used as the active electrodes. A reference electrode was attached to the skin near the eye and a ground electrode was clipped into the skin of the tail. ERG recordings were collected using RETI scan system (Roland Consult, Wiesbaden, Germany) at a sampling rate of $2 \mathrm{kHz}$. The amplitudes of a-wave and b-wave were measured from the average of three responses by a set of three flashes of stimulation, using two flash intensities, 3.0 and $10.0 \mathrm{~cd} \mathrm{~s} / \mathrm{m}^{2}$. In each group, ERG recording was performed on eight eyes.
H\&E staining. Eyes were fixed with $4 \%$ formalin overnight, embedded in paraffin and cut into $3 \mu \mathrm{m}$ vertical slices. Sections were washed and treated with hematoxylin buffer for $10 \mathrm{~min}$ at room temperature. Then, sections were rinsed in deionized water and dipped in $1 \%$ eosin solution for $15 \mathrm{~s}$. After rehydrating in alcohol gradient, slices were washed again and mounted. Histological analyses of retinal tissues were observed under a microscope (Leica DM4000, Wetzlar, Germany) for calculating cell nuclei in the GCL from six microscopic fields per eye and at least six eyes were used for analysis. Images were processed with ImageJ software (public domain, imagej.nih.gov/ij).

BV2 cell culture and treatment. Murine BV2 microglial cells (Kunming Institute of Zoology, Chinese Academy of Sciences, Beijing, China) were maintained in DMEM supplemented with $10 \% \mathrm{FBS}$, streptomycin $(50 \mathrm{mg} / \mathrm{ml})$ and penicillin $(50$ $\mathrm{U} / \mathrm{l})$. LPS $(100 \mathrm{ng} / \mathrm{ml})$ or $\mathrm{H}_{2} \mathrm{O}_{2}(300 \mu \mathrm{M})$ were added to the cultures, respectively. The cells were identified using Iba- 1 and CD11b, the classical markers for microglia. ${ }^{18,19}$ For treatment, BV2 cells were pre-treated with either Nec-1 (20 and $40 \mu \mathrm{M}$ ) or vehicle $2 \mathrm{~h}$ before LPS or $\mathrm{H}_{2} \mathrm{O}_{2}$ stimulation. Twenty-four hours later, cells were collected for further investigation. For cell transfection, TLR2-siRNA, TLR4siRNA constructs were diluted in buffer (Lipofectamine transfection reagent, Invitrogen Inc., Carlsbad, CA, USA) and transfected in culture medium of BV2 cells at a working concentration of $100 \mathrm{nM}$ for $24 \mathrm{~h}$ before $\mathrm{H}_{2} \mathrm{O}_{2}$ stimulation, and the transfection efficacy was evaluated by western blot. The sequences for TLR2-siRNA and TLR4-siRNA are 5'-CCAATCTCACAAATTTACA-3', and 5'-CAATCTGACGA ACCTAGTA-3', respectively.

Immunofluorescence assay on retinal flat mounts. Eyes were removed and fixed in 4\% paraformaldehyde (PFA) for 30 min. Retinae were harvested and incubated with primary and secondary antibodies, and then washed in PBST and flat mounted. The primary antibodies include anti-lba-1 antibody (Wako Chemicals, Osaka, Japan), anti-RIP1 antibody (BD Bio-Sciences, Bedford, MA, USA), anti-CD11b, anti-TNF- $\alpha$ and anti-RIP3 antibodies (Abcam, Cambridge, MA, USA), anti-TLR2 antibody (Genway Biotech Inc., San Diego, CA, USA), and anti-TLR4 antibody (Santa Cruz Biotechnology Inc., Santa Cruz, CA, USA) and anti-CCL2 antibody (Novus Biologicals, Littleton, CO, USA). The secondary antibodies include donkey anti-rabbit IgG H\&L (Alexa Fluor 488, 555), donkey anti-mouse IgG H\&L (Alexa Fluor 405, 555) and donkey anti-goat IgG H\&L (Alexa Fluor 555, 647) antibodies (Abcam). Primary antibodies were diluted at 1:100 for usage, and the secondary antibodies were applied at $1: 1000$ in dilution. In each group, six retinal flat mounts were performed and observed under a confocal microscope (Carl Zeiss LSM710, Jena, Germany).

TUNEL assay. After being immersed in 4\% PFA fixative, retinae were removed and embedded in optimal cutting temperature compound (Sakura Finetechnical Co., Tokyo, Japan) overnight and sectioned through the whole eye. TUNEL staining (In Situ Cell Death Detection Kit, Fluorescein; Roche, Indianapolis, IN, USA) was performed according to the manufacturer's instructions. For double staining of TUNEL and cleaved-caspase-3, sections were first stained with rabbit anti-cleaved-caspase-3 antibody and then incubated with donkey anti-rabbit IgG H\&L (Alexa Fluor 555) secondary antibody together with TUNEL staining. Slides were then mounted with antifade medium containing DAPI and photographed using a confocal microscope (Carl Zeiss LSM710).

Western blotting. Retinal and cellular protein were harvested and homogenized in lysis buffer (RIPA, Biocolors, Shanghai, China) containing protease and phosphatase inhibitor mini tablets (Thermo Fisher Scientific, no. 88668; Waltham, MA, USA). The protein concentration was determined with bicinchoninic acid assay. Western blotting was performed as described previously. Briefly, $30 \mu \mathrm{g}$ of protein was loaded per lane on a $10 \%$ SDS-PAGE. The membrane blots were saturated with $5 \% \mathrm{BSA}$ in PBST for $1 \mathrm{~h}$ at room temperature and then incubated overnight at $4{ }^{\circ} \mathrm{C}$ with antibodies against TNF- $\alpha$, RIP3 and MLKL (1:1000, Abcam), CCL2 (1: 1000, Novus Biologicals), RIP1 (1:1000, BD Bio-Sciences), IL-6 (1: 1000, R\&D Systems, Minneapolis, MN, USA), IL- $1 \beta$, IL-23, IFN- $\gamma$ ( $1: 500$, Santa Cruz, CA, USA) and $\beta$-actin (1:2000, Abcam) were used. For the assessment of RIP1 phosphorylation, lysates were first immunoprecipitated with anti-RIP1 antibody overnight. The immunocomplexes were then analyzed by western blotting with antiRIP1 antibody and anti-phosphotyrosine antibody (p-Ser, $1: 200$, Santa Cruz). The gray intensity of proteins was measured using Image $\mathrm{J}$ software (US National Institutes of Health). 
Real-time PCR. The total RNA of the retinae were extracted with TRIzol (Invitrogen) and converted into first-strand cDNA using random hexamer primers and the Reverse Transcriptase Superscript II Kit (Invitrogen). PCR was performed in a total volume of $20 \mu \mathrm{l}$ containing $2 \mu \mathrm{l}$ of CDNA, $10 \mu \mathrm{l}$ of $2 \times$ SYBR Premix Ex Taq and $10 \mu \mathrm{mol} / / \mathrm{l}$ of the primer pairs. The primers were tnf- $\alpha$ fwd $5^{\prime}$ GAGGCCAAGCCCTGGTATG-3' and rev 5'-CGGGCCGATTGATCTCAGC-3', and ccl2 fwd 5'-TAAAAACCTGGATCGGAACCAAA-3' and rev 5'-GCATTAGCTTC AGATTTACGGGT-3'. Gapdh was used as a reference gene. The PCR amplification protocols consisted of $95^{\circ} \mathrm{C}$ for $30 \mathrm{~s}$ and up to 40 cycles of $95^{\circ} \mathrm{C}$ for $5 \mathrm{~s}$ and $60^{\circ} \mathrm{C}$ for $34 \mathrm{~s}$ according to the manufacturer's instructions.

ELISA assay. BV2 cells were seeded on 12-well plates (Corning Inc., Corning, $\mathrm{NY}, \mathrm{USA}$ ) and cultured in serum-free DMEM overnight. Cells were then pre-treated with $20 \mu \mathrm{M} \mathrm{Nec}-1$ or vehicle $2 \mathrm{~h}$ before $\mathrm{H}_{2} \mathrm{O}_{2}$ stimulation $(300 \mu \mathrm{M})$ for $24 \mathrm{~h}$. The supernatants of BV2 cells were then collected, and TNF- $\alpha$ and CCL2 levels were detected using mouse ELISA kits (R\&D Systems) according to the manufacturer's instructions.

Statistical analysis. Representative results are shown in the figures. The sample size is chosen as generally required for basic research. For immunofluorescence staining, TUNEL assay and H\&E staining, at least six retinae in each group were used, and 6-8 images were randomly captured from each retina for analysis. For other experiments including western blotting, aPCR and ELISA, at least four retinae per animal group and three independent cultured cells per group were used and these experiments were independently repeated at least three times. Samples (mice and cell cultures) are randomly assigned to experimental groups. Cell number calculation and data analysis were performed by two blind researchers. Data are presented as mean \pm S.E.M., and were analyzed statistically using one-way ANOVA or two-tailed Student's $t$-test. $P$-values $<0.05$ were considered statistically significant.

\section{Conflict of Interest}

The authors declare no conflict of interest.

Acknowledgements. This study was supported by National Natural Science Foundation of China to XLL (no. 81630022, 81470646.) and by the Fundamental Research Funds for the Central Universities to $\mathrm{CH}$ (no. 17ykzd27).

1. Heneka MT, Carson MJ, El Khoury J, Landreth GE, Brosseron F, Feinstein DL et al. Neuroinflammation in Alzheimer's disease. Lancet Neurol 2015; 14: 388-405.

2. Tansey MG, Goldberg MS. Neuroinflammation in Parkinson's disease: its role in neuronal death and implications for therapeutic intervention. Neurobiol Dis 2010; 37: 510-518.

3. Donoso LA, Kim D, Frost A, Callahan A, Hageman G. The role of inflammation in the pathogenesis of age-related macular degeneration. Surv Ophthalmol 2006; 51: 137-152.

4. Carlson NG, Wieggel WA, Chen J, Bacchi A, Rogers SW, Gahring LC. Inflammatory cytokines IL-1 alpha, IL-1 beta, IL-6, and TNF-alpha impart neuroprotection to an excitotoxin through distinct pathways. J Immunol (Baltimore, MD : 1950) 1999; 163: 3963-3968.

5. Conductier G, Blondeau N, Guyon A, Nahon JL, Rovere C. The role of monocyte chemoattractant protein MCP1/CCL2 in neuroinflammatory diseases. J Neuroimmunol 2010; 224: 93-100.

6. Langmann T. Microglia activation in retinal degeneration. J Leukocyte Biol 2007; 81: $1345-1351$

7. Streit WJ, Mrak RE, Griffin WS. Microglia and neuroinflammation: a pathological perspective. J Neuroinflammation 2004; 1: 14.

8. Perry VH, Nicoll JA, Holmes C. Microglia in neurodegenerative disease. Nat Rev Neurol 2010; 6: 193-201.

9. Perry VH, Holmes C. Microglial priming in neurodegenerative disease. Nat Rev Neurol 2014; 10: $217-224$

10. Pasparakis M, Vandenabeele P. Necroptosis and its role in inflammation. Nature 2015; 517 311-320.

11. Kaczmarek A, Vandenabeele P, Krysko DV. Necroptosis: the release of damage-associated molecular patterns and its physiological relevance. Immunity 2013; 38: 209-223.

12. Lau A, Wang S, Jiang J, Haig A, Pavlosky A, Linkermann A et al. RIPK3-mediated necroptosis promotes donor kidney inflammatory injury and reduces allograft survival. $A m \mathrm{~J}$ Transplant 2013; 13: 2805-2818.
13. Pavlosky A, Lau A, Su Y, Lian D, Huang X, Yin Z et al. RIPK3-mediated necroptosis regulates cardiac allograft rejection. Am J Transplant 2014; 14: 1778-1790.

14. Gunther C, Martini E, Wittkopf N, Amann K, Weigmann B, Neumann H et al. Caspase-8 regulates TNF-alpha-induced epithelial necroptosis and terminal ileitis. Nature 2011; 477: 335-339.

15. Bonnet MC, Preukschat D, Welz PS, van Loo G, Ermolaeva MA, Bloch W et al. The adapto protein FADD protects epidermal keratinocytes from necroptosis in vivo and prevents skin inflammation. Immunity 2011; 35: 572-582.

16. Linkermann A, Green DR. Necroptosis. New Engl J Med 2014; 370: 455-465.

17. Lenzlinger PM, Morganti-Kossmann MC, Laurer HL, Mclntosh TK. The duality of the inflammatory response to traumatic brain injury. Mol Neurobiol 2001; 24: 169-181.

18. Mosher KI, Andres RH, Fukuhara T, Bieri G, Hasegawa-Moriyama M, He Y et al. Neural progenitor cells regulate microglia functions and activity. Nat Neurosci 2012; 15: 1485-1487.

19. Koriyama Y, Nakayama Y, Matsugo S, Sugitani K, Ogai K, Takadera T et al. Antiinflammatory effects of lipoic acid through inhibition of GSK-3beta in lipopolysaccharideinduced BV-2 microglial cells. Neurosci Res 2013; 77: 87-96.

20. Nakazawa T, Takahashi H, Nishijima K, Shimura M, Fuse N, Tamai M et al. Pitavastatin prevents NMDA-induced retinal ganglion cell death by suppressing leukocyte recruitment. $J$ Neurochem 2007; 100: 1018-1031.

21. Seya T, Shime H, Takaki H, Azuma M, Oshiumi H, Matsumoto M. TLR3/TICAM-1 signaling in tumor cell RIP3-dependent necroptosis. Oncoimmunology 2012; 1: 917-923.

22. Takeuchi O, Akira S. Pattern recognition receptors and inflammation. Cell 2010; 140 $805-820$

23. Saijo K, Glass CK. Microglial cell origin and phenotypes in health and disease. Nat Rev Immunol 2011; 11: 775-787.

24. Cunningham $\mathrm{C}$. Microglia and neurodegeneration: the role of systemic inflammation. Glia 2013; 61: 71-90.

25. Jennewein C, Karl S, Baumann B, Micheau O, Debatin KM, Fulda S. Identification of a novel pro-apoptotic role of NF-kappaB in the regulation of TRAIL- and CD95-mediated apoptosis of glioblastoma cells. Oncogene 2012; 31: 1468-1474.

26. Kiyota T, Gendelman HE, Weir RA, Higgins EE, Zhang G, Jain M. CCL2 affects betaamyloidosis and progressive neurocognitive dysfunction in a mouse model of Alzheimer's disease. Neurobiol Aging 2013; 34: 1060-1068.

27. Sakurai E, Anand A, Ambati BK, van Rooijen N, Ambati J. Macrophage depletion inhibits experimental choroidal neovascularization. Invest Ophthalmol Vis Sci 2003; 44 3578-3585.

28. Bennett ML, Bennett FC, Liddelow SA, Ajami B, Zamanian JL, Fernhoff NB et al. New tools for studying microglia in the mouse and human CNS. Proc Natl Acad Sci USA 2016; 113: E1738-E1746.

29. Moriwaki K, Chan FK. Necroptosis-independent signaling by the RIP kinases in inflammation. Cell Mol Life Sci 2016; 73: 2325-2334.

30. Ofengeim D, Yuan J. Regulation of RIP1 kinase signalling at the crossroads of inflammation and cell death. Nat Rev Mol Cell Biol 2013; 14: 727-736.

31. Festjens N, Vanden Berghe T, Cornelis S, Vandenabeele P. RIP1, a kinase on the crossroads of a cell's decision to live or die. Cell Death Differ 2007; 14: 400-410.

32. Degterev A, Huang Z, Boyce M, Li Y, Jagtap P, Mizushima $\mathrm{N}$ et al. Chemical inhibitor of nonapoptotic cell death with therapeutic potential for ischemic brain injury. Nat Chem Biol 2005; 1: 112-119.

33. He S, Liang $Y$, Shao F, Wang $X$. Toll-like receptors activate programmed necrosis in macrophages through a receptor-interacting kinase-3-mediated pathway. Proc Natl Acad Sci USA 2011; 108: 20054-20059.

34. Fernandez-Lizarbe S, Montesinos J, Guerri C. Ethanol induces TLR4/TLR2 association, triggering an inflammatory response in microglial cells. J Neurochem 2013; 126: 261-273.

35. Li Y, Zhang F, Nagai N, Tang Z, Zhang S, Scotney P et al. VEGF-B inhibits apoptosis via VEGFR-1-mediated suppression of the expression of $\mathrm{BH} 3$-only protein genes in mice and rats. J Clin Invest 2008; 118: 913-923.

$(9)(9)$ This work is licensed under a Creative Commons Attribution-NonCommercial-NoDerivs 4.0 International License. The images or other third party material in this article are included in the article's Creative Commons license, unless indicated otherwise in the credit line; if the material is not included under the Creative Commons license, users will need to obtain permission from the license holder to reproduce the material. To view a copy of this license, visit http://creativecommons.org/licenses/by-nc-nd/4.0/

(C) The Author(s) 2018 